\title{
The Structure of Developmental Variation in Early Childhood
}

\author{
Benjamin A. Stenhaug ${ }^{1}$, Nilam Ram², and Michael C. Frank ${ }^{2}$ \\ ${ }^{1}$ The Graduate School of Education \\ Stanford University \\ ${ }^{2}$ Department of Psychology \\ Stanford University
}

\begin{abstract}
Author Note
The research reported here was supported by the Institute of Education Sciences, U.S. Department of Education, through Grant R305B140009 to the Board of Trustees of the Leland Stanford Junior University. The opinions expressed are those of the author and do not represent views of the Institute or the U.S. Department of Education. Correspondence concerning this article should be addressed to Benjamin A. Stenhaug at benastenhaug@gmail.com
\end{abstract}




\begin{abstract}
Do children's abilities develop in tandem or on their own separate timetables? Piaget proposed that development proceeded globally through stages; more recent theories view development as more modular with different abilities developing independently and on different time-scales. The developmental differentiation hypothesis suggests that the structure of a child's development is unitary early in infancy but becomes more complex with age. Despite an abundance of theoretical interest in this question, there is little empirical work on the macrostructure of developmental changes in early childhood. We investigate this structure using two large datasets of parent-reported developmental milestones. Applying item response theory models, we find that variation in development across infancy and early childhood is multidimensional. Consistent with the differentiation hypothesis, differences among older children are better described by higher-dimensional models. In addition, in longitudinal data, we find that, within-person changes in underlying abilities are highly coupled early in life but their coupling decreases by age 12 months. Our work provides a model-based method for linking holistic descriptions of early development to basic theoretical questions about the nature of change in childhood.
\end{abstract}

\title{
Significance Statement
}

How do children vary? Do some children develop globally faster or slower than others, or is variation more discrete (e.g., in motor or language skill)? Variation between children has often been assumed to be unifactorial or multifactorial without formal evaluation. Our work here uses psychometric modeling and big-data approaches to evaluate this dimensionality empirically. We find evidence for multidimensionality, and further evidence that this dimensionality increases with age. This work implies that measures of developmental variation should move beyond assumptions that differences and progression of children's development can be represented as a homogenous process, and toward multi-dimensional representations of within-person change. 


\section{The Structure of Developmental Variation in Early Childhood}

\section{Introduction}

How do young children grow and change? Is child development a single unified process or a host of different processes, each with its own constraints and timescale? Piaget famously proposed a stage theory in which many seemingly distinct mental processes developed in concert through a common set of operational stages (1). In contrast, modern theories propose that there are different facets of children's mental life and that these facets each develop on their own timetable (2). And attesting to a folk theory of developmental multi-dimensionality, the grandmother of one author was known to assert that "children either walk early or else they talk early."

A theoretical and practical understanding of how children grow and change provides the underpinning for parents', teachers', and health professionals' efforts to observe and facilitate children's development. However, the process of assessing children's developmental status critically depends on our assumptions about the structure of developmental change - in particular, whether there is a single unified process that can be measured through tracking of developmental milestones. Global assessment of developmental status via a series of binary milestones (e.g., "Can your child walk at least ten steps unassisted?") is both a standard feature of pediatrician visits (3) and a gold standard for assessing children's developmental status in the research and intervention communities (4-6). In such assessments, which are typically but not always conducted via parent report, developmental progress is often treated as, per Piaget, an amalgam of motoric, cognitive, and language achievements. Thus, most instruments implicitly assume a unifactorial model (although some also provide subscale scores; 4).

The dimensionality of children's variation is not necessarily constant - it could itself change developmentally. Indeed, some early work argued that a single, general ability factor transitions into multiple factors between 8 and 18 years of age (7). We refer to the general idea of an increase in the factor structure of developmental variation as "the 
differentiation hypothesis." The differentiation hypothesis was later extended to the differentiation-dedifferentiation hypothesis, which holds that abilities separate during the first half of the life span and then collapse back together later in life $(8,9)$. Being a within-person hypothesis, the strongest evidence for the (de)differentiation hypothesis requires longitudinal data so as to identify the expanding or collapsing of factors within individuals (10).

There is mixed evidence of differentiation in adolescence $(11,12)$ and mixed evidence of de-differentiation in adulthood $(13,14)$, but the differentiation hypothesis has not been examined in early childhood and has typically not been evaluated as a within-person hypothesis (see 10 as an exception). Availability of appropriate data is likely a limiting factor. Although large-scale datasets are everywhere (15), few focus on early childhood (cf. 16, 17). Those that do include infants and young children do not provide a holistic picture of development. Comprehensive empirical examinations require longitudinal data that tracks how many children progress through many milestones, with limited missingness and reasonably short intervals between assessments. The big-data obtained via mobile apps open new opportunities for studying how development manifests in real-world settings, although data quality often remains a challenge (18).

This paper leverages two datasets - survey data and mobile app data, both provided by parents as their children developed - to explore the structure of developmental variation in early childhood. In the cross-sectional survey data, middle-class Mexican parents of children between 2 and 55 months old $(\mathrm{N}=1,946)$ provided comprehensive reports about whether or not their children had achieved 414 developmental milestones. In the longitudinal mobile app data, over 20,000 parents repeatedly reported on their child's achievement of collections of age-specific developmental milestones as part of their use of a mobile application that provided child development related video content. The app used milestone reports as a method for assessing children's progress and serving appropriate content. By using survey data in conjunction with app data, we leverage the structure of 
each data source to provide empirical information about the structure of developmental variation in early childhood.

The foundations of our inquiry are built from principles of measurement/testing and computational data science. We use psychometric models to instantiate specific hypotheses about psychological structure and assess how well various structures fit the data. In particular, we leverage item response theory (IRT) models (first developed by Educational Testing Service to measure students' academic performance; 19), to describe the structure of developmental variation in early childhood. We also leverage the sheer size of these newly available data through use of cross-validation and construction of hold-out data sets that facilitate iterative exploration and confirmation of the structural and differentiation hypotheses. In doing so, we propel forward the integration of developmental and data science now afforded by the arrival and curation of big data from the deployment of mobile technologies.

We conducted two studies using these datasets. Study 1 used a collection of item response models with different numbers of factors to describe the structure of between-child developmental variation in the survey data. As evidence for multidimensionality, we found that models with more factors performed better according to out-of-sample accuracy. And, consistent with the differentiation hypothesis, models with more factors provided more complete descriptions of the differences among older children.

At its core, the differentiation hypothesis is a theory about how individual children develop. In particular, the differentiation hypothesis posits that within-child covariation between developmental factors will be high very early on and decrease as children age. Between-child differences, like those examined in Study 1, are often examined in relation to the age-related differentiation hypothesis, but risk falling prey to the ecological fallacy: seeming age differences in structure might instead indicate differences in developmental timing or selection. Thus, in Study 2, we leveraged additional longitudinal data that more specifically supports examination of how the covariance between developmental factors 
changes within-person over time. Specifically, using a 2-factor model from Study 1-where the 1st factor serves as a broad indicator of physical achievement and the 2nd factor serves as a broad indicator of linguistic achieve - we model both how children develop over time and how the coupling between gains on each of the two factors changes with age. Here, we find strong evidence of within-person differentiation hypothesis, with the high early covariation between the two factors systematically decreasing across early childhood.

Taking the two studies together, our work makes three contributions. First, we describe the between-child multidimensional structure of developmental variation in early child. Second, we find that the dimensionality of this variation increases with age. Third, we find that within-child covariation between factors decreases during a child's first year (i.e., evidence for the differentiation hypothesis).

\section{Developmental Milestone Data}

As children develop, they achieve - and sometimes move through - many milestones, including shaking objects, crawling, pointing, taking turns, and drawing shapes. While developing and norming a set of web- and smartphone-based parenting applications, Kinedu, Inc. obtained data on how and when many children achieved a wide variety of developmental milestones. Study 1 leverages survey data obtained from 1,946 Mexican parents reporting on whether or not their children, age 2 to 55 months, had achieved 414 milestones: 180 physical milestones (e.g., child can go from sitting to kneeling), 100 cognitive milestones (e.g., child can find objects on the floor), 75 linguistic milestones (e.g., child can say four words), and 59 social-emotional milestones (e.g., child shows concern for a crying friend). As shown in Figure 1, by age 1 month, most children had achieved about 50 of the developmental milestones; by age 24 months, most children had achieved about 300 of the developmental milestones. These data, binary responses (has not or has achieved) to multiple items of different difficulty, have the same structure as item response data commonly obtained in educational settings. For example, when taking standardized 
tests in school, students' responses to long batteries of questions graded as correct or incorrect are used to track achievement and learning. Accordingly, we use the psychometric models developed in educational measurement to study the structure of students' academic abilities to study the structure of children's achievement of developmental milestones across early childhood.

\section{Item Response Models}

Item response models provide a robust framework for assessing the structure of an instrument, the relative difficulty of each item, and respondents' level of performance on the abilities or latent factors measured by the instrument. Here, we use two-parameter logistic (2PL) item response models to assess the structure of children's development.

Let $i=1, \ldots, I$ represent the distinct children and $j=1, \ldots, J$ the developmental milestones. The item response data is stored in a matrix where element $y_{i j}$ denotes if the $i$ th child has or has not achieved the $j$ th developmental milestone as reported by their parent/guardian. Each model represents the $i$ th child's development using $m$ latent factors by $\boldsymbol{\theta}_{i}=\left(\theta_{1}, \ldots, \theta_{m}\right)$. The $j$ th milestone's discriminations (i.e. slopes) $\boldsymbol{a}_{\boldsymbol{j}}=\left(a_{1}, \ldots, a_{m}\right)$ capture the latent factor loadings onto that milestone. We fit five $2 \mathrm{PL}$ models where a child's development is represented by $m=1, m=2, m=3, m=4$ and $m=5$ latent factors (20). Hereafter, we, for example, refer to a 2PL model with $m=4$ latent factors as a $4 \mathrm{~F}$ model. According to the 2PL model, the probability of child $i$ having achieved developmental milestone $j$ is

$$
P\left(y_{i j}=1 \mid \boldsymbol{\theta}_{\boldsymbol{i}}, \boldsymbol{a}_{\boldsymbol{j}}, b_{j}\right)=\sigma\left(\boldsymbol{a}_{j}^{\top} \boldsymbol{\theta}_{\boldsymbol{i}}+b_{j}\right)
$$

where $b_{j}$ is the milestone easiness (i.e. intercept) and $\sigma(x)=\frac{e^{x}}{e^{x}+1}$ is the standard logistic function.

By comparing models with varying numbers of factors, we learn about the dimensionality of the construct measured by the instrument. If, for example, models with 
numerous factors fit the data better than a $1 \mathrm{~F}$ model, then across-child comparisons are best made in a multidimensional space.

We compare these item response models to a simple baseline model that predicts achievement status based on milestone and age in months. The baseline model always predicts the modal response for a particular milestone and age. For example, parents report that $64 \%$ of children age 16 -months can identify animals by their sounds; the baseline model assumes that all 16-month-olds have reached this milestone. This baseline aims to represent predictive performance in practical settings, absent the use of item response models.

\section{Study 1: The Structure of Developmental Variation Across Individuals}

We measure a model's fit to the data's structure based on how well it predicts data previously unknown to the model (i.e., out-of-sample data) (21). In particular, we use cross-validation to compare item response models with varying numbers of factors. We partition the milestone responses into 8 folds. For each model, we implement the following process. For each fold (the out-of-sample fold), we estimate milestone and child development parameters using milestone responses from the other 7 folds (the in-sample folds). We use these parameters to make predictions for responses in the out-of-sample fold. We aggregate performance from when each fold is out-of-sample to calculate the overall out-of-sample accuracy of a model. To estimate explained variance, we also extracted the proportion of variance explained by the model from a single fit of each model to the full dataset (i.e., without cross-validation).

\section{Developmental Variation is Multidimensional}

Results from fitting the five different item response models are shown in Table 1. While the unidimensional model (1F: $88.8 \%$ accuracy) fit better than the baseline (86.9\%), the multidimensional models $(2 \mathrm{~F}$ to $5 \mathrm{~F})$ all fit the data better $(>89.3 \%)$. The $5 \mathrm{~F}$ model provided $89.8 \%$ out-of-sample accuracy, and thus (of the models fit) provided the best 
predictive performance. The $5 \mathrm{~F}$ model explained less variance than the $4 \mathrm{~F}$ model, however, likely indicating that further increases in dimensionality would not yield better fit to data.

Each of the models uncovered a consistent structure where linguistic milestones have the greatest loadings on the 1st factor (i.e., linguistic milestone discriminate well between children high and low on the 1st factor; Figure 22. For the 2F-5F models, physical milestones tend to have the greatest loadings on the 2nd factor. Additional factors capture variation from fewer milestones as seen by the majority of discriminations being relatively close to zero.

\section{Older Children's Milestones are More Accurately Described by Higher-Dimensional Models}

The between-person version of the differentiation hypothesis suggests that the abilities of older children have a higher-dimensional structure. To examine the possibility of age-related differences in dimensionality we partitioned the milestone data into roughly 1-year age groups: age 2 to 11 months, 12 to 23 months, 24 to 35 months, and 36 to 47 months and 48 to 55 months, with the upper two age groups consolidated together to obtain similar sample size in each partition. We then calculated the gain over the $1 \mathrm{~F}$ model for each of the $2 \mathrm{~F}$ to $5 \mathrm{~F}$ models in each of the age-group partitions. We calculated gain as the proportion of the distance between the $1 \mathrm{~F}$ model's performance and $100 \%$ that the model achieves. For example, for 24 to 35-month-olds, the 5F model has $88.4 \%$ accuracy as compared to the $1 \mathrm{~F}$ model which has $87.2 \%$ accuracy. The gain of the $5 \mathrm{~F}$ model over the $1 \mathrm{~F}$ model, then, is $\frac{88.4 \%-87.2 \%}{100 \%-87.2 \%}=9.4 \%$. The top panel of Figure 3 shows the gain of each higher-dimensional model over the $1 \mathrm{~F}$ model. The $5 \mathrm{~F}$ model performs best for each bin, and also performs particularly well for the older age groups. The relative increase in gain for the 5F model (and other multi-dimensional models) at older ages is consistent with the differentiation hypothesis. 


\section{Differences Between Old Children Are Better Described by Higher-Dimensional Models}

Leveraging the sheer size of the data to push beyond evaluation of predictive performance of global models in different age groups, we also examined relative performance within each age-group partition separately. In particular, we conducted the 8-fold cross-validation procedure again within each partition of the data. The bottom panel of Figure 3 shows the gain for each of the higher dimensional models over the $1 \mathrm{~F}$ model for each age group fit separately. Using these age-partitioned models, we find similar but perhaps somewhat stronger evidence for the differentiation hypothesis: The gain of higher dimensional models is modest for the youngest age group and substantially higher for older age groups.

\section{Study 2: The Dimensionality of Within-Child Variability Increases Across Development}

Drawing conclusions about within-child processes using between-child data risks committing a version of the ecological fallacy. The ecological fallacy typically cautions against making individual-level claims from group-level data (22). In the case of the differentiation hypothesis, the "group" is the child and the "individual" is each of the timepoints that the child is measured at. Patterns across children are not necessarily indicative of patterns across individual childrens' timepoints. Therefore, the best assessment of the differentiation hypothesis requires longitudinal data.

We leverage the use of Kinedu, Inc.'s application as our source of such longitudinal data. As part of using the app, parents are asked to respond to 20-50 milestones about their child every few months. As with many mobile apps, usage is non-uniform, such that many users use the app once or only occasionally, while a smaller number used it frequently. We focus here on the most frequent users of the app, as their relatively dense data provide the best opportunity to test hypotheses about within-child change. We 
selected data from children with at least 6 timepoints, where a timepoint contains at least 5 responses to each of the 4 milestone categories. We also focus here on children between 2 and 18 months old because parents typically use the app when their child is in this age range and so there were few children with dense data in older age ranges.

A previous test of the de-differentiation hypothesis using longitudinal data examined deviations from individual developmental pathways to identify how these deviations related to one another (10). We adapt this method, which was developed for continuous outcomes, to factor scores estimated from binary outcomes. In brief, the goal of this method is to ask whether local deviations from the child's own developmental trajectory are related to one another ("coupling"). For example, if a child surges ahead on motor milestones in a particular month, will they also surge ahead on linguistic milestones?

Computing coupling of this type involves five distinct steps (Figure 4 visualizes the first four). The first step was to develop an appropriate measurement model. Because the survey data is complete and likely to be higher quality, we chose to use the survey data to develop this model. To ensure that the model was well-calibrated for the app data, we filtered to children 24 months of age or younger and removed a small number of children with an unexpected number of milestones complete for their age (e.g., a 9-month-old with more than 350 milestones complete). A 2F model offers good fit and an interpretable factor structure for this population. The 1st and 2nd factors capture $35 \%$ and $20 \%$ of the survey data variance, respectively (an additional factor would explain only $5 \%$ more variance). These factors appear to load more heavily on physical milestones vs. linguistic milestones, respectively. Although models with more factors show the same differentiation effect described below (see SI), the finding is easiest to conceptualize in a two-factor space.

The second step was to estimate factor scores for each child-timepoint in the app data according to the parsimonious $2 \mathrm{~F}$ model fit to the survey data. As expected, these factor scores are highly correlated with age. In the third step, we separately estimated a quadratic developmental pathway for each child. In the fourth step, we calculate the 
deviation from the child's developmental pathway for each timepoint.

The fifth and final step was to fit a mixed-effects model to estimate coupling across the age span for all children. The model predicts the deviation for one factors using the other factor's deviation (and interactions with age), while controlling for differences between individual children using random effects (following 10). Larger coefficients on factor deviation indicate greater coupling, such that, for example, if at a particular time point the child increases on factor 1 they are more likely to increase on factor 2 .

In developing our method, we used an exploratory sample of 600 children $(4,734$ timepoints, $M=7.9$ timepoints/child). We then replicated the procedure using two additional samples of 600 children (we leave some data unanalyzed for future exploration). In all three samples, we find strong evidence for the differentiation hypothesis. For example, a 1-unit deviation from factor 2's developmental pathway is associated with at least a 0.75 -unit deviation in the same direction from factor 1's developmental path at 2 months old. And, this association decreases to less than a 0.15 -unit deviation for children older than 12 months old. We find similar results when inverting the factors and considering the association between deviations from factor 1's developmental pathway on deviations from factor 2's developmental path. These results are depicted in Figure 5 .

In sum, this finding gives strong evidence that, within individual children, local deviations from the child's own developmental growth trajectory begin quite coupled but appear to decouple by around 18 months. Put another way, when a young baby grows especially quickly or slowly they tend to do so on all relevant dimensions (at least within our milestone set). In contrast, a toddler's language can surge forward (or hang back) without coordinated changes in motor development. Thus, we find evidence for the differentiation hypothesis within individual children. 


\section{General Discussion}

Is child development a single unified process or a host of different processes? Stage theories assume synchronization in developmental changes across distinct domains like language, social-emotional development, and cognition (1). In contrast, more modern modular theories tend to assume that particular aspects of development proceed "on their own schedule" $(3,23)$. Here, inspired by psychometric studies of age-related changes in cognition, we explored this issue through the lens of individual variation. Our premise was that understanding the nature of variation in developmental milestones could help shed light on whether children's developmental change covaries across domains within a single factor and whether developmental variation splits into multiple factors as children grow older.

With the emergence of data science techniques, our use of newly available data is a specific instance of a general pattern: Larger data enables more precise measurements, which can be used to test and refine theories. Although theorists have speculated about developmental differentiation, to our knowledge the differentiation hypothesis had not previously been tested using large-scale data. Our contributions came from leveraging data made newly available due to parents' use of a mobile app. Parents, who were looking to understand and support their child's development, answered hundreds of binary milestone questions about their child. As a result, we were able to explore and test the differentiation hypothesis in early childhood by fitting a variety of psychometric models to this data.

We modeled the structure of age-related variation between children and identified data-driven dimensions in this variation. These appeared to map to some extent onto classic domains of development, for example by loading more heavily on motor or language milestones. It is important to remember, however, that by nature of our analyses, our results describe differences rather than commonalities between individuals. Because we leverage individual variability, our models are not designed to detect the operation of mechanisms that are consistent across individuals. Despite the variation we observed and 
quantified, many common mechanisms (from statistical learning to motor skill learning) likely support developmental change across all individuals. The individual differences in milestones that we observe then might simply be differences in learning rates across individuals.

Our study has several limitations that should inform future work. First, we relied on parent report, which can have significant biases and limitations, especially in its precision regarding capacities that are difficult to observe (e.g., cognitive abilities: 16, 24). Second, our data come from very specific populations (Study 1: middle- and upper-class Mexican parents whose children were in group care; Study 2: an unknown but largely Brazil, US, and Mexico-based group of users of a developmental mobile application) and hence caution is warranted in generalizing to specific populations. Third, our results are with regard to child development as defined by the Kinedu app milestone set. These milestones provide a global picture of observable developmental changes, but they do not necessarily carve development at its joints. Further, the factor discovery methods we use reflect the statistical structure of the observed data rather than the structure of any latent variables internal to the child (25-27).

In the end, our goal is a first step in the direction of leveraging large datasets to better understand child development. Our approach was to combine multiple data sources and develop novel methods which together allowed us to explore theories of child development. In doing so, we found multidimensionality in the variation between children and support for the development differentiation hypothesis both across and within children. Our hope is that this work encourages others to continue to advance developmental theory by taking advantage of opportunities provided by new, larger data sources.

\section{Materials and Methods}

All data were provided by Kinedu, Inc. A pre-registration, code, and data are publicly available at https://osf.io/5426p/. We deviated from the pre-registration for two 
reasons. First, we had not planned to use longitudinal data but came to appreciate the importance of these data for examination of differentiation as a within-person phenomenon. Second, we had failed to appreciate the degree to which lack of milestone overlap across ages (i.e., planned missingness for older children to younger milestones and vice versa) jeopardize making valid cross-age comparisons. In our Supplemental Information, we report on an additional dataset that we preregistered the intent to analyze, but eventually deemed unsuitable for these reasons.

\section{Survey Data}

The survey data used in Study 1 was collected by Kinedu, Inc. during the development of their mobile application. The surveys were completed by middle- and upper-class Mexican parents whose children were in group care. The raw survey data contains complete (i.e., no missingness) responses to all 414 milestones for 2,023 children. We filtered out children younger than 2 months old due to concerns about data quality (e.g., 40\% of 0- and 1-month-olds had surprisingly achieved at least 80 milestones). The analysis data for our examination of dimensionality (Study 1) included data on whether or not 1,946 children age 2 to 55 months had achieved 414 cognitive, linguistic, physical and social-emotional milestones.

\section{App Data}

The app data focused on in Study 2 was collected by Kinedu, Inc. during normal operations of their development mobile application. In particular, the app asks parents to report on age-appropriate milestones for their child every few months. We considered data collected between the beginning of the current 414 milestones (12/01/2017) and the start of data analysis for this part of the project (12/16/2020). Our goal was to create a longitudinal dataset. We created "timepoints" based on milestones responses that were collected within a 5-day window for each child. We subset down to timepoints where a significant number of milestones were responded to-specifically, we required at least 5 
responses to each of the 4 milestone categories for a total of at least 20 responses. To target the app's typical users, we considered only timepoints when the child was at least 2 months old and less than 18 months old. We did so both to mitigate any floor or ceiling effects and to reduce any potential selection bias that might result from parents who elect to use the app when their child is older than was typical. Finally, so as to have sufficiently longitudinal data for each child, we considered only children with at least 6 such timepoints. The result of these pre-processing steps was the "app data" containing 21,861 unique children and 177,934 total timepoints. We used only 1,800 of these children in the form of three 600 child samples. Each sample was drawn randomly. The first sample (the exploratory sample) was used while developing methods. The second and third samples (the replication samples) were used to test the validity of results from the exploratory sample.

\section{Study 1: Dimensionality of Developmental Milestones in Early Childhood (Cross-validation of Item Response Models)}

To examine the structure and dimensionality of children's achievement of developmental milestones, we compared the relative fit of item response models with between 1 and 5 factors. Model comparison in IRT typically uses information criterion such as AIC and BIC (28). However, these methods are not guaranteed to work with modest sample sizes or when the models are misspecified (29). Instead, we implemented a version of cross-validation for item response models with varying numbers of factors (30). The steps of our cross-validation procedure are as follows. We began by partitioning the data into 8 folds. We stratified randomization by child so that each fold contained approximately one-eighth of each child's milestone responses. In other words, each child was split into 8 "sub-children" and each fold contained exactly one of these sub-children. We refer to the fold hidden from the data as out-of-sample and the other 7 folds as in-sample. Holding each of the 8 folds out one at a time, we then conduct the following steps for each model. 
We first fit the model to the in-sample data. According to an m-dimensional 2PL model, the probability of a child having achieved a developmental milestone is

$$
P\left(y_{i j}=1 \mid \boldsymbol{\theta}_{i}, \boldsymbol{a}_{j}, b_{j}\right)=\sigma\left(\boldsymbol{a}_{j}^{\top} \boldsymbol{\theta}_{i}+b_{j}\right)
$$

where the $j$ th milestone's discriminations (i.e. slopes) $\boldsymbol{a}_{j}=\left(a_{1}, \ldots, a_{m}\right)$ capture the latent factor loadings onto that milestone; $b_{j}$ is the milestone easiness (i.e. intercept); and $\sigma(x)=\frac{e^{x}}{e^{x}+1}$ is the standard logistic function. We stabilize the model by using a Normal $(0$, 3) prior on all $b_{j}$ values. We do not use a prior on so as to not interfere with interpretations of dimensionality. As is common for item response models, we used marginal maximum likelihood estimation (MMLE), which estimates milestone parameters but not child parameters (31). Higher dimensional models require slightly different estimation methods so the full latent factor space is adequately considered: For models with 3, 4, or 5 factors, we used quasi-Monte Carlo EM estimation (32); for models with 1 or 2 factors we used the standard EM algorithm (33). To allow for correlations in the factor structure, we estimated milestone parameters under an oblimin rotation (34).

Second, we estimated factor scores for each child using the in-sample data. We estimated factor scores using expected a posterior (EAP) with a m-dimensional normal prior as calculated by Gauss-Hermite quadrature (35). Third, we use the milestone parameters and factor scores estimated using the in-sample data to predict the probability of each out-of-sample milestone response.

Going through these steps for each fold resulted in a predicted probability for each milestone response. We evaluated each model's fit by simply considering these predictions' accuracy (correct if the predicted probability was greater than $50 \%$ and the milestone was achieved or if the predicted probability was less than $50 \%$ and the milestone was not achieved). We compared these models to a simple baseline model that makes predictions based on a child's age (in months) and the milestone. For example, parents reported that $64 \%$ of 16 -month-olds can identify animals by their sounds, so the baseline model predicts that all 16-month-olds have reached this milestone (when fewer than $50 \%$ of children at 
that age had not achieved the milestone, the baseline model predicts that none of the children at that age in months have achieved the milestone).

We examined the relationship between age and dimensionality using two techniques. Both techniques involve measuring the relative gain of a higher dimensional model relative to a $1 \mathrm{~F}$ model. Letting $a c c_{\text {model }}$ represent the out-of-sample accuracy from a higher-dimensional model and $a c c_{1 F}$ represent the out-of-sample accuracy from a $1 \mathrm{~F}$ model, we calculated gain as $a c c_{\text {model }}-a c c_{1 F} 1-a c c_{1 F}$. Intuitively, gain can be thought of as the higher dimensional model's percent of possible improvement in accuracy over the $1 \mathrm{~F}$ model. Our first technique was break down each model's performance by age group so that we could calculate the gain by age group. This involved estimating 40 models $(5$ models $\cdot 8$ folds $)$. If the structure of developmental variation changes with age, then it may be suboptimal to estimate models based on all children. Accordingly, our second technique was to estimate each model separately for each of the age groups. This involved estimating 200 models (5models $\cdot 8$ folds $\cdot 5$ agegroups). We then calculated the gain for each model in each age group. For some age groups, nearly all (or no) children have accomplished some of the milestones. To prevent these milestones from destabilizing model estimation, we filtered out milestones from an age group's data when more than $97.5 \%$ or fewer than $2.5 \%$ of children had achieved the milestone. Figure 3 shows results from both techniques, with the top panel corresponding to the first and the bottom panel corresponding to the second.

\section{Study 2: Testing the Differentiation Hypothesis Using Longitudinal Item Response Data}

The differentiation hypothesis suggests that the structure of children's abilities expands with age. In Study 2, we use the longitudinal app data to test this hypothesis. To do so, we adapt methods developed in examination of dedifferentiation of cognitive abilities in old age (10) to analysis of factor scores obtained from multidimensional item response 
models of the milestone data obtained on up to 21 occasions as children moved through early childhood. As detailed below, we use multilevel model models of change to examine if and how age moderates the extent of within-person coupling in the occasion-to-occasion changes in factors underlying milestone achievement (estimated using item response models), after controlling for individuals' general developmental gains. Our expectation is that, separate from the long-term developmental trends, within-person variations on 2 underlying ability factors will be tightly coupled (i.e., dedifferentiated) at earlier ages and that the extent of coupling will decrease towards zero as children get older and the underlying factors differentiate. Analysis proceeded in five steps: (1) develop a measurement model, (2) estimate factor scores, (3) model longer-term developmental trends, (4) extract deviations from longer-term developmental trends, and (5) examine age-related differences in within-person coupling of factor scores. The first four steps are shown in Figure 4. The fifth step is shown in Figure 5.

\section{Step 1: Develop a Measurement Model}

Our first analytic task was to obtain measurement invariant factor scores from the longitudinal milestone achievement data. Taking a relatively conservative approach, we use a filtered version of the survey data. We did so because (a) the app data skews younger than the survey data and (b) the survey data started to show ceiling effects at 24 months old (see Figure 1). In particular, we filter the survey data to children between 1 and 24 months old (931 of the 2,023 children). We also filter outlier children by considering only children with more than $15+8 \cdot$ ageinmonths and less than $100+14 \cdot$ ageinmonths milestones achieved (909 out of 931 children). We measure age in months where a month is 30.3 days. We used a $2 \mathrm{~F}$ model because our goal was to understand the relationship between main factors (a $2 \mathrm{~F}$ model explained $55 \%$ of the variance; a $3 \mathrm{~F}$ model explained

$60 \%$ of the variance). We estimated milestone parameters by using MMLE to fit this $2 \mathrm{~F}$ 
model, which specifies the probability of child $i$ having accomplished milestone $j$ as

$$
P\left(y_{i j}=1\right)=\sigma\left(a_{j 1} \theta_{i 1}+a_{j 2} \theta_{i 2}+b_{j}\right)
$$

where $a_{j 1}$ and $a_{j 2}$ are milestone $j$ 's slope with respect to factor 1 and 2 , respectively; $\theta_{i 1}$ and $\theta_{i 2}$ are child 's factor 1 and factor 2 scores, respectively; and $b_{j}$ is milestone $j$ 's easiness. As is common when fitting a multidimensional item response model with a limited sample size, we used priors to stabilize the model. In particular, we used a $\operatorname{Normal}(0,3)$ prior on $b_{j}$ and a $\operatorname{Lognormal}(0,0.5)$ prior on $a_{j 1}$ and $a_{j 2}$ values. Given that the factors were high correlated, an oblique rotation was required. Accordingly, we estimated milestone parameters under an oblimin rotation (34).

\section{Step 2: Estimate Factor Scores}

The model parameters were tabulated and used to estimate factor scores using EAP with a 2-dimensional normal prior as calculated by Gauss-Hermite quadrature (35), for each of the children on each occasion in the longitudinal app data. Importantly, these factor scores capture within-person changes in two measurement invariant ability factors underlying children's achievement of cognitive, linguistic, physical and social-emotional milestones across early childhood, age 2 to 55 months.

\section{Step 3: Model Longer-term Developmental Trends and Step 4: Extract Deviations}

Having obtained estimated factor scores for each factor on each occasion for each child, we proceeded to model the systematic age-related changes in each factors. Separately for each child, the longitudinal factor scores for the first factor were modeled as

$$
\theta_{1 t i}=B_{10 i}+B_{11 i}\left(a g e_{t i}\right)+B_{12 i}\left(a g e_{t i}^{2}\right)+e \theta_{1 t i}
$$

where the estimated factor score for child $i$ on occasion $t$, was modeled as a trajectory defined by a person-specific intercept $B_{10 i}$, a person-specific linear slope, $B_{11 i}$, a 
person-specific quadratic slope, $B_{12 i}$. Deviations from the age trajectory, then are captured by the person- and occasion-specific residuals, $e \theta_{1 t i}$. In parallel, the longitudinal factor scores for the second factor were modeled as

$$
\theta_{2 t i}=B_{20 i}+B_{21 i}\left(a g e_{t i}\right)+B_{22 i}\left(a g e_{t i}^{2}\right)+e \theta_{1 t i}
$$

where the estimated factor score for child $i$ on occasion $t, \theta_{1 t i}$ was modeled as a trajectory defined by a person-specific intercept $B_{20 i}$, a person-specific linear slope, $B_{21 i}$, a

person-specific quadratic slope, $B_{22 i}$. Deviations from the age trajectory, then are captured by the person- and occasion-specific residuals, $e \theta_{2 t i}$. Models were fit to each child's repeated measures (between 6 and 21 occasions) using the $\operatorname{lm}$ function in $\mathrm{R}$ (embedded in a data selection and parameter tabulation loop).

The third panels of Figure 4 shows the developmental pathway for a single child. While these developmental trajectories are interesting in their own right, our intent here is to remove these trends in order to examine the extent to which the occasion-to-occasion changes in the factor scores are coupled with each other beyond the expected gains captured by the long-term trends. Thus, of greatest interest here are the residuals, $e \theta_{1 t i}$ and $e \theta_{2 t i}$. The residuals for the same child are shown in the fourth panel of Figure 4 . These residuals allow us to examine the differentiation hypothesis as a within-person phenomenon separate from the "normative" gains in children's cognitive, linguistic, physical, and social-emotional abilities. Any coupling in these "left over" occasion-to-occasion changes is then attributable to unobserved occasion-specific common-causes (that are not directly attributable to age).

\section{Step 5: Examine Age-related Differences in Within-person Coupling of Factor Scores}

The differentiation hypothesis suggests that occasion-specific changes in children's underlying ability factors will be highly coupled in infancy and then differentiate with age. Said differently, factors that tend to travel together over time (high within-person coupling) 
early on will become increasingly independent at older ages (lower and lower within-person coupling). We test this hypothesis using a multilevel model that explicitly articulates if and how the extent of within-person coupling between the repeated measures data-which we detrended in a very conservative way in the previous step to obtain $e \theta_{1 t i}$ and $e \theta_{2 t i}$ - differs as a function of children's age. Specifically, we modeled the detrended factor scores as

$$
e \theta_{2 t i}=\alpha_{1 i}\left(e \theta_{1 t i}\right)+\alpha_{2}\left(e \theta_{1 t i} * a g e_{t i}\right)+\alpha_{3}\left(e \theta_{1 t i} * a g e_{t i}^{2}\right)+r_{t i}
$$

where $\alpha_{1 i}$ is a person-specific coefficient that indicates the extent of within-person coupling in child $i$ 's repeated measures and $r_{t i}$ are residual errors that are assumed normally distributed (note that there is no person-specific intercept because, by definition the outcome variable has a mean of zero) The within-person coupling coefficients are modeled as $\alpha_{1 i}=\gamma_{10}+u_{1 i}$

$$
\alpha_{2 i}=\gamma_{20}
$$

$\alpha_{3 i}=\gamma_{30}$ where the age gradient in the extent of coupling is described by an intercept parameter, $\gamma_{10}$, that indicates the expected coupling for the prototypical child at age 0 months (centering age), and linear and quadratic slope parameters, $\gamma_{20}$ and $\gamma_{30}$. Person-specific deviations from the average coupling are captured by $u_{1 i}$, which is assumed normally distributed with mean of zero and standard deviation, $\sigma_{u 1}$. For completeness, a parallel model where $e \theta_{1 t}$ was used as the outcome variable and $e \theta_{2 t}$ as the predictor was also fit to the data.

\section{Replication Strategy}

We executed these steps on an exploratory sample and two replication samples, which each contained 600 randomly selected children. Prior to running analysis on the exploratory sample, we examined scatterplots of $e \theta_{1 t i}$ and $e \theta_{2 t i}$ and noted a few $(<10)$ observations that might potentially serve as influential outliers. Taking a conservative stance, we removed observations where the absolute value of either $e \theta_{1 t i}$ or $e \theta_{2 t i}$ was greater than 1.5 (fewer than $0.2 \%$ of timepoints). This rule (as with the entire analysis) was 
captured in a function that we then executed without examination on the two replication samples. Models were fit using the lmer package in $\mathrm{R}$, with restricted maximum likelihood estimation and incomplete data treated as usual under missing at random assumptions (36).

\section{Acknowledgments}

We thank Kinedu, Inc. for financial support and data sharing. We thank George Kachergis, Alex Carstensen, Ben Domingue, and Ann Weber for comments on early versions of this paper. The research reported here was also supported by the Institute of Education Sciences, U.S. Department of Education, through Grant R305B140009 to the Board of Trustees of the Leland Stanford Junior University. The opinions expressed are those of the author and do not represent views of the Institute or the U.S. Department of Education. 


\section{References}

1. Flavell JH (1963) The developmental psychology of jean piaget.

2. Gelman, R. and B. Baillargeon (1983) Review of some Piagetian concepts. In Handbook of Child Psychology (Volume II) (Wiley, New York). pp. 167-230.

3. Sheldrick RC, et al. (2019) Establishing new norms for developmental milestones. Pediatrics 144(6).

4. Bayley N (2009) Bayley-III: Bayley scales of infant and toddler development (Giunti OS).

5. Bricker D, et al. (1999) Ages and stages questionnaire. Paul H Brookes: Baltimore.

6. McCoy DC, Gonzalez K, Jones S (2019) Preschool self-regulation and preacademic skills as mediators of the long-term impacts of an early intervention. Child development 90(5):1544-1558.

7. Garrett HE (1946) A developmental theory of intelligence. American Psychologist $1(9): 372$.

8. Lienert G, Crott H (1964) Studies on the factor structure of intelligence in children, adolescents, and adults. Vita Humana:147-163.

9. Breit M, Brunner M, Preckel F (2020) General intelligence and specific cognitive abilities in adolescence: Tests of age differentiation, ability differentiation, and their interaction in two large samples. Developmental psychology 56(2):364.

10. Hülür G, Ram N, Willis SL, Schaie KW, Gerstorf D (2015) Cognitive dedifferentiation with increasing age and proximity of death: Within-person evidence from the seattle longitudinal study. Psychology and Aging 30(2):311.

11. Juan-Espinosa M, Cuevas L, Escorial S, Garcia LF (2006) Testing the indifferentiation hypothesis during childhood, adolescence, and adulthood. The 
Journal of genetic psychology 167(1):5-15.

12. Shing YL, Lindenberger U, Diamond A, Li S-C, Davidson MC (2010) Memory maintenance and inhibitory control differentiate from early childhood to adolescence. Developmental Neuropsychology 35(6):679-697.

13. Hartung J, Doebler P, Schroeders U, Wilhelm O (2018) Dedifferentiation and differentiation of intelligence in adults across age and years of education. Intelligence 69:37-49.

14. Li S-C, et al. (2004) Transformations in the couplings among intellectual abilities and constituent cognitive processes across the life span. Psychological science $15(3): 155-163$.

15. Tsai C-W, Lai C-F, Chao H-C, Vasilakos AV (2015) Big data analytics: A survey. Journal of Big data 2(1):1-32.

16. Frank MC, Braginsky M, Yurovsky D, Marchman VA (2021) Variability and consistency in early language learning: The wordbank project (MIT Press).

17. Mindell JA, Sedmak R, Boyle JT, Butler R, Williamson AA (2016) Sleep well!: A pilot study of an education campaign to improve sleep of socioeconomically disadvantaged children. Journal of clinical sleep medicine 12(12):1593-1599.

18. Milne-Ives M, Velthoven MH van, Meinert E (2020) Mobile apps for real-world evidence in health care. Journal of the American Medical Informatics Association 27(6):976-980.

19. Lord FM (1980) Applications of item response theory to practical testing problems (Routledge).

20. Chalmers RP (2012) Mirt: A multidimensional item response theory package for the r environment. Journal of Statistical Software 48(6):1-29.

21. Stone M (1974) Cross-validatory choice and assessment of statistical predictions. 
Journal of the Royal Statistical Society: Series B (Methodological) 36(2):111-133.

22. Piantadosi S, Byar DP, Green SB (1988) The ecological fallacy. American journal of epidemiology 127(5):893-904.

23. Spelke ES, Breinlinger K, Macomber J, Jacobson K (1992) Origins of knowledge. Psychological review 99(4):605.

24. Feldman HM, et al. (2000) Measurement properties of the MacArthur communicative development inventories at ages one and two years. Child development 71(2):310-322.

25. Bork R van, Epskamp S, Rhemtulla M, Borsboom D, Maas HL van der (2017) What is the p-factor of psychopathology? Some risks of general factor modeling. Theory \&3 Psychology 27(6):759-773.

26. Maraun M (2003) Myths and confusions: Psychometrics and the latent variable model. Unpublished Manuscript Retrieved from http://www sfu ca/ maraun/myths-and-confusions html.

27. Van Der Maas HL, et al. (2006) A dynamical model of general intelligence: The positive manifold of intelligence by mutualism. Psychological review 113(4):842.

28. Maydeu-Olivares A (2013) Goodness-of-fit assessment of item response theory models. Measurement: Interdisciplinary Research and Perspectives 11(3):71-101.

29. McDonald RP, Mok MM-C (1995) Goodness of fit in item response models. Multivariate Behavioral Research 30(1):23-40.

30. Bergner Y, et al. (2012) Model-based collaborative filtering analysis of student response data: Machine-learning item response theory. International Educational Data Mining Society.

31. Baker FB, Kim S-H (2004) Item response theory: Parameter estimation techniques (CRC Press). 
32. Jank W (2005) Quasi-monte carlo sampling to improve the efficiency of monte carlo EM. Computational statistics $\&$ data analysis 48(4):685-701.

33. Bock RD, Aitkin M (1981) Marginal maximum likelihood estimation of item parameters: Application of an EM algorithm. Psychometrika 46(4):443-459.

34. Jennrich RI, Sampson P (1966) Rotation for simple loadings. Psychometrika $31(3): 313-323$.

35. Embretson SE, Reise SP (2013) Item response theory (Psychology Press).

36. Bates D (2005) Fitting linear mixed models in $\mathrm{r}$. $R$ news 5(1):27-30. 


\section{Table 1}

Model performance as measured by out-of-sample accuracy. Higher-dimensional models perform better.

\begin{tabular}{lcc}
\hline Model & Out-of-sample Accuracy & Proportion of Variance \\
\hline $5 \mathrm{~F}$ & $89.8 \%$ & $73.0 \%$ \\
$4 \mathrm{~F}$ & $89.7 \%$ & $74.5 \%$ \\
$3 \mathrm{~F}$ & $89.5 \%$ & $73.3 \%$ \\
$2 \mathrm{~F}$ & $89.3 \%$ & $66.1 \%$ \\
$1 \mathrm{~F}$ & $88.8 \%$ & $60.1 \%$ \\
Baseline & $86.9 \%$ & - \\
\hline
\end{tabular}




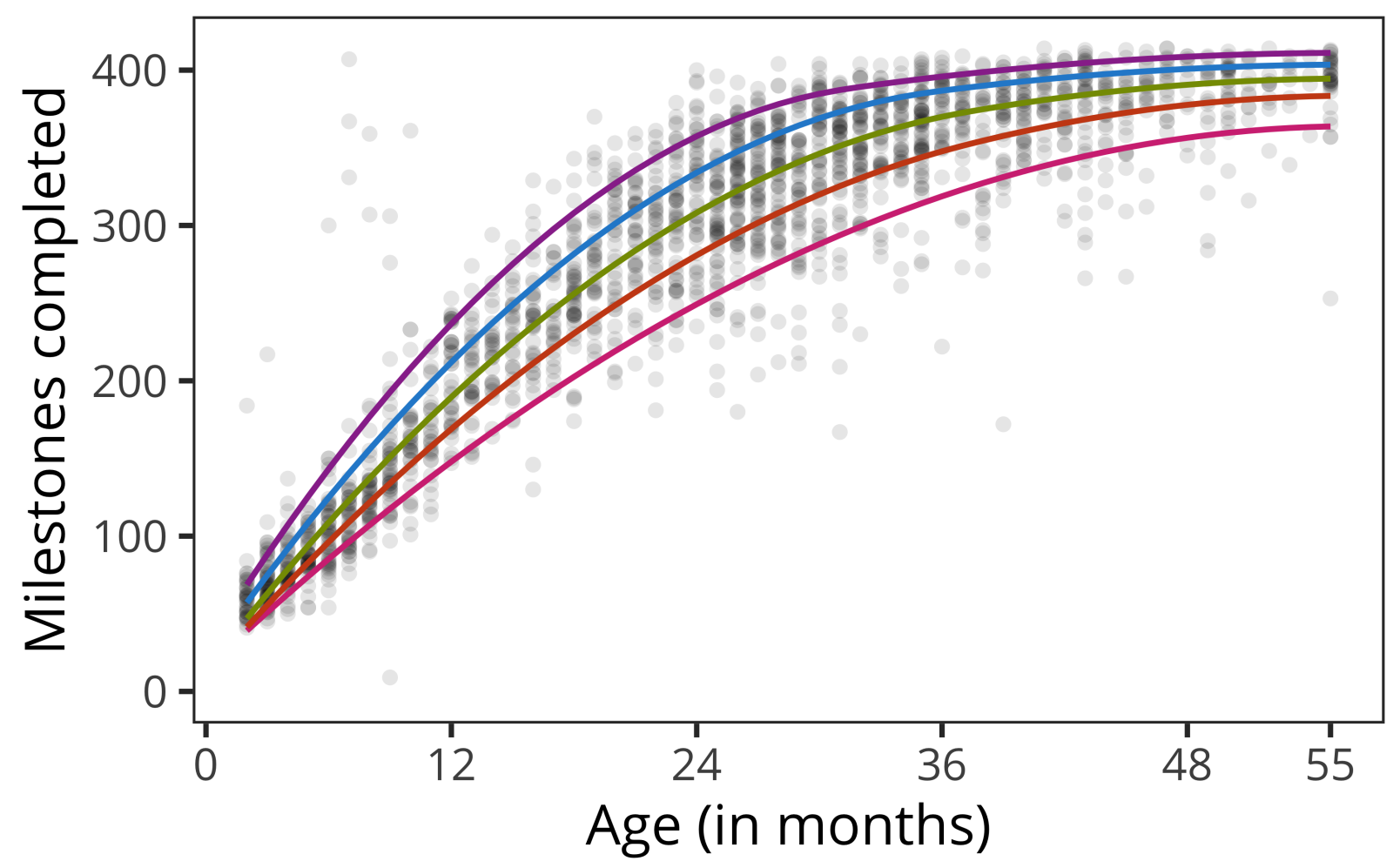

Figure 1

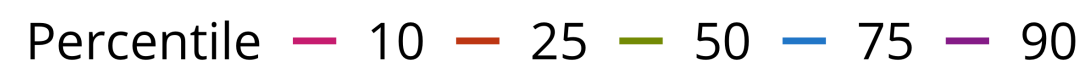

Number of the 414 milestones completed by age with percentile curves; data are from the survey reported in Study 1. Points represent individual children. 




Figure 2

Discrimination

Distribution of discrimination parameters for the factors of the $1 F, 2 F, 3 F, 4 F$, and $5 F$ models. Columns of subplots show models, rows show factors, and distributions are the density of discrimination parameter estimates, colored by broad milestone categories. In each of the models, linguistic milestones load heavily on the 1st factor. Additional factors tend to be composed of other milestone categories - for example, physical milestones tend to load heavily on the 2nd factor. As expected, the typical discrimination decreases for later factors. Arrows track the location of two milestones, crawling (physical) and saying at least 4 words (linguistic), across each of the factors. 


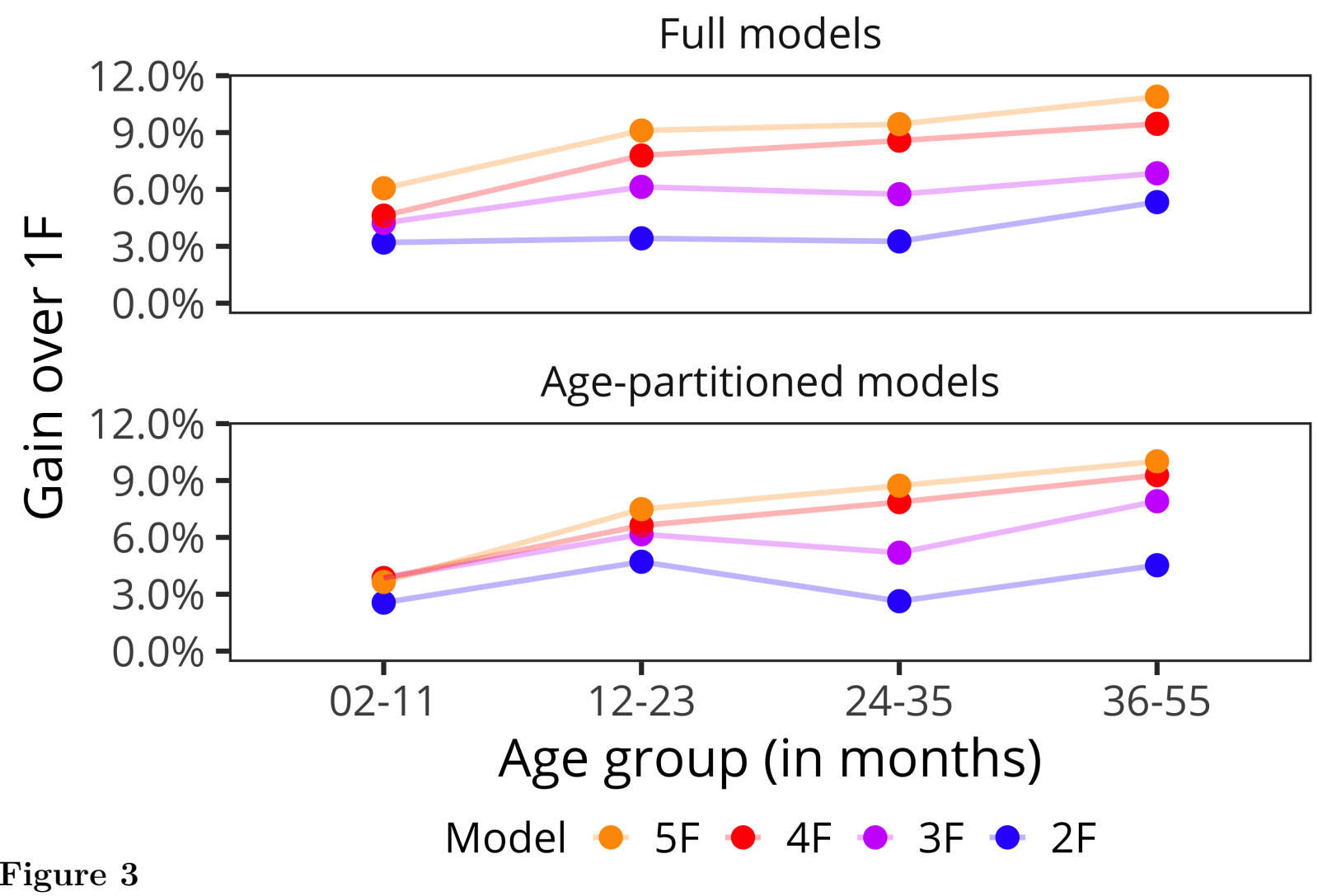

Gain of higher-dimensional models over $1 F$ model. Gain is defined as the proportion of the distance between the $1 F$ model's performance and $100 \%$ that the model achieves. The top panel shows that when each model is fit to the full dataset, higher-dimensional models perform particularly well for older age groups. The bottom panel shows that when each model is fit separately to each age group, higher-dimensional models perform particularly well for older age groups. 

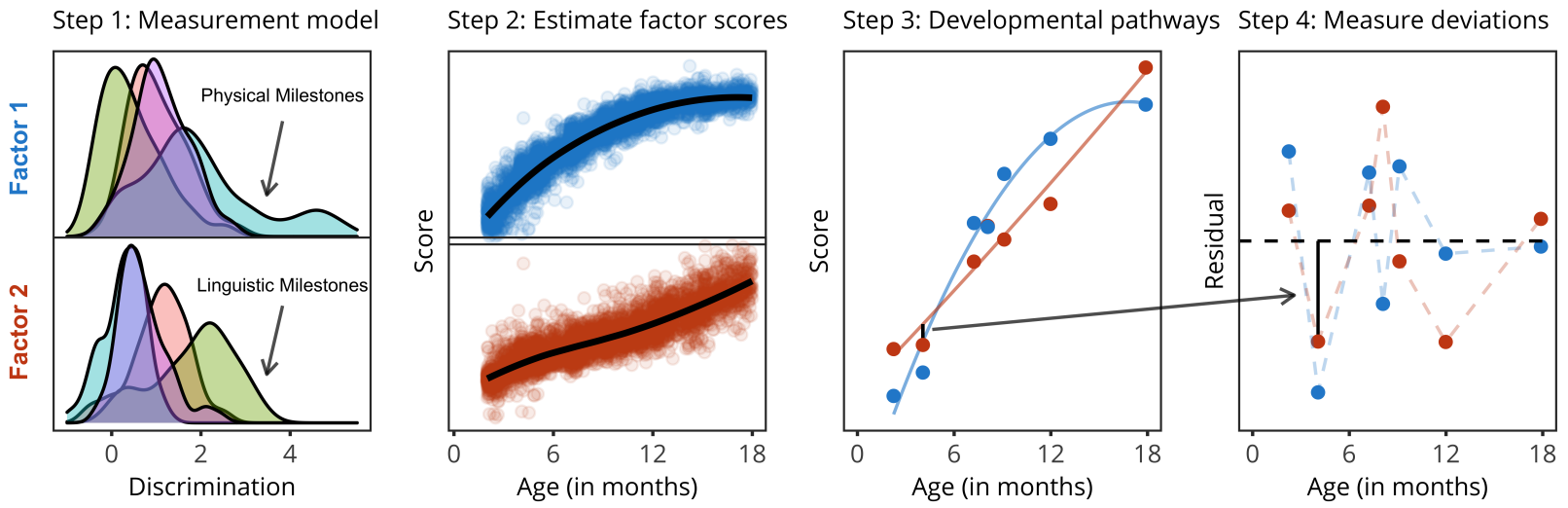

Figure 4

Each panel corresponds to a step from Study 2. In the first step, we used the survey data to develop a measurement model. The first factor is mainly physical and the second factor is mainly linguistic. In the second step, we used the measurement model to estimate factor scores for each child-timepoint in the app data. As expected, both factors are highly associated with age. In the third step, we modeled longer-term developmental trends separately for each child. Here, we illustrate this step by showing the trends for a single child. In the fourth step, we extract the deviations (i.e., residuals) from the developmental trends. Here, we show the deviations (i.e., residuals) for that same child. These deviations allow us to examine age-related differences in within-person coupling of factor scores. 


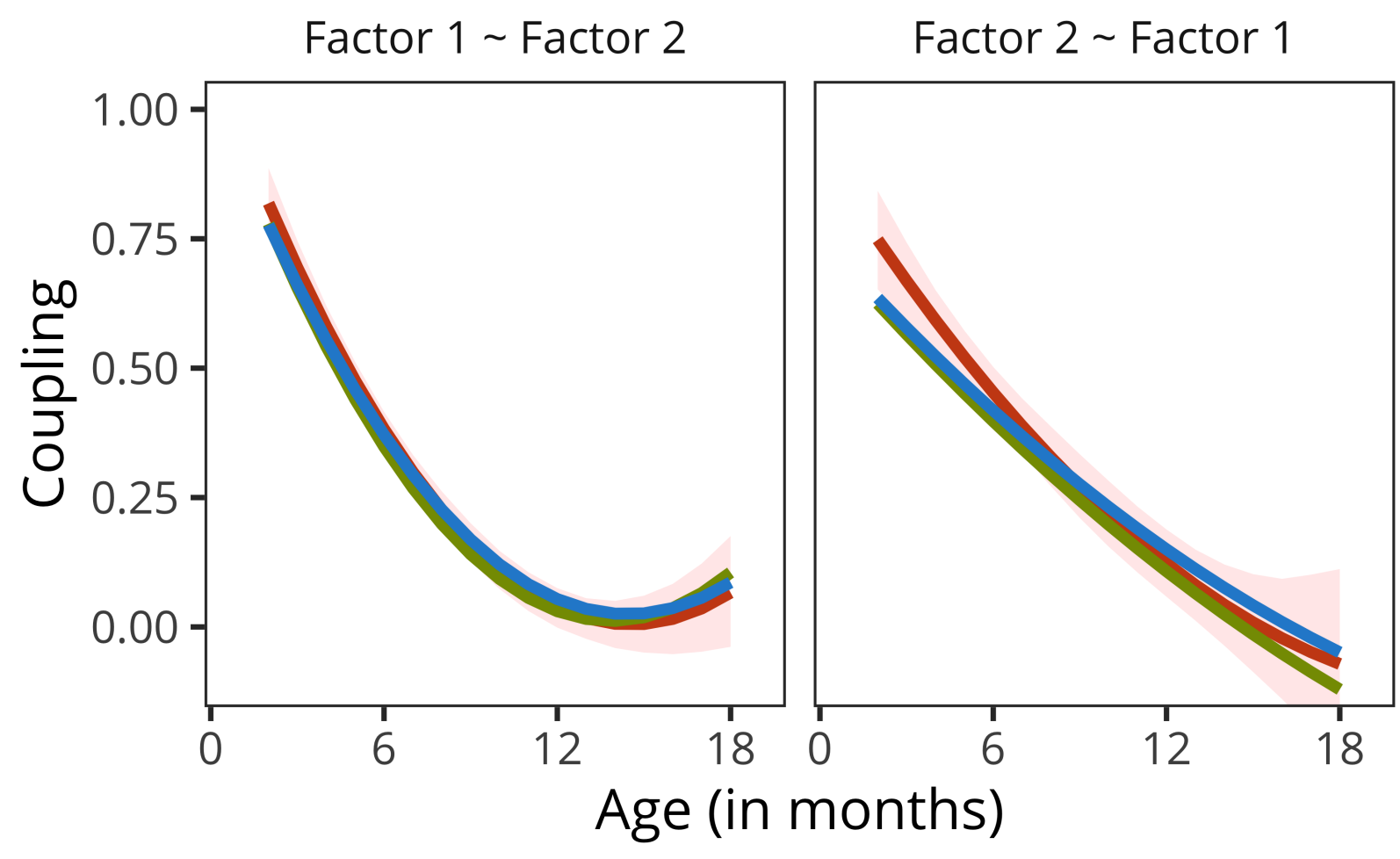

Figure 5

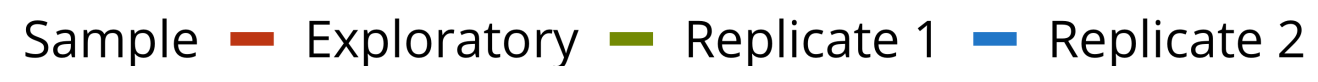

Coupling parameters from 2 to 18 months old. Association between 1-unit deviation from the developmental pathway for one factor with deviation from the other factor's developmental path. Left panel shows factor 1 as the dependent variable and factor 2 as the independent variable. Right panel is the inverse. As the differentiation hypothesis suggests, we find decreasing coupling over the age span. Red shading is $95 \%$ confidence interval for exploratory sample as calculated by 1000 bootstrapped simulations. 